\title{
OCCURRENCE OF METRONIDAZOLE AND IMIPENEM RESISTANCE AMONG BACTEROIDES FRAGILIS GROUP CLINICAL ISOLATES IN HUNGARY*
}

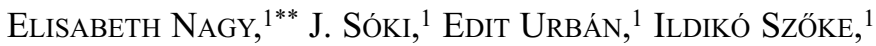 \\ ELEONÓRA FODOR ${ }^{1}$ and R. EdWARds ${ }^{2}$ \\ ${ }^{1}$ Institute of Clinical Microbiology, Faculty of Medicine, University of Szeged, Szeged, Hungary \\ ${ }^{2}$ Division of Microbiology and PHLS Laboratory, University Hospital, Queen's Medical Centre, \\ Nottingham, UK
}

(Received: August 31, 2000; accepted: October 5, 2000)

\begin{abstract}
During the period between 1987 and 1997, various surveillances of the antibiotic resistance of B. fragilis group isolates revealed that practically all the isolates tested were susceptible to imipenem, metronidazole and chloramphenicol; very few isolates $(2.5 \%)$ exhibited resistance to amoxicillin/clavulanic acid. However, similarly as in some southern European countries, the percentages of the isolates that were resistant to ampicillin, tetracycline and clindamycin were high throughout this period, and the resistance to cefoxitin increased from $6 \%$ to $16 \%$. In 2000 , isolates with intermediate or high resistance to imipenem and isolates with increased MICs to metronidazole were emerging among the clinical isolates of B. fragilis. The presence of the cfiA gene was demonstrated by PCR in 7 of 242 isolates (2.9\%); 2 of them with high MICs to carbapenems harboured the IS942 element immediately upstream of the resistance genes. In the $2 \mathrm{~B}$. fragilis isolates with increased MICs to metronidazole, the nim gene could be detected by PCR. The IS1186 element was found in these isolates upregulating the metronidazole resistance gene.
\end{abstract}

Keywords: Bacteroides - imipenem - metronidazole - PCR - IS elements

\section{INTRODUCTION}

In view of their high isolation rate from clinical specimens, the species of the genus Bacteroides sensu stricto are the most important of the anaerobic pathogens. Bacteroides fragilis occurs in such samples most frequently, outnumbering the related species by a factor of ten [9]. The infections, which are endogenous and often associated with facultative anaerobic species, include intra-abdominal, intra-pelvic, lung and brain abscesses, peritonitis and sepsis. The pathogenicity factors of $B$. fragilis include the capsular polysaccharide, which is a potent inducer of soft-tissue abscesses, the ability to bind to fibronectin and collagen-I, an iron uptake mechanism, lipopolysaccharide and the production of a metalloprotease toxin which causes diarrhoea [12]. Apart from their clinical significance, they are important and the most abundant $\left(10^{11}\right.$ cells/g faeces) bacterial constituents of the colon, the distal small and the large bowel, where they contribute to the physiological activities of the

\footnotetext{
*Dedicated to Professor Lajos Ferenczy on the occasion of his 70th birthday.

**Corresponding author; e-mail: nagye@mlab.szote.u-szeged.hu
} 
intestine, e.g. in the degradation of residual biopolymers, the biotransformation of bile acids, the production of vitamin $\mathrm{K}$ and the prevention of pathogenic organisms from colonization [14]. The third important feature of the genus Bacteroides is that its species are among the most resistant bacteria of all anaerobes and have the most numerous resistance mechanisms to antimicrobial agents [21].

Resistance against $\beta$-lactams is mediated mainly by $\beta$-lactamases, which are of two important types. The members of the first group are cephalosporinases belonging in group 2e in the Bush classification scheme [4]; they have molecular masses of 30-40 $\mathrm{kDa}$, are inhibited by regular $\beta$-lactamase inhibitors such as clavulanic acid and sulbactam, hydrolyse cephalosporins rather than penicillins, and do not hydrolyse cefoxitin or carbapenems; their genes are termed cepA $[6,21]$. The members of the second group are metallo- $\beta$-lactamases that have molecular masses of $25-33 \mathrm{kDa}$, belong in Bush functional group 3, are inhibited by EDTA, but not by regular $\beta$-lactamase inhibitors, require $\mathrm{Zn}^{2+}$ for their activity, and are capable of hydrolysing cephamycins or carbapenems; their sequenced genes are named cfiA or ccrA [6,21]. Clindamycin resistance in Bacteroides strains is mediated by a macrolide-lincomycin-streptogramin (MLS) mechanism, which causes the methylation of specific adenosine residue(s) on the 23S rRNA. The gene encoding this type of resistance, ermF, resides on regular transposons which may be carried on plasmids or chromosomal conjugative elements [21]. It is on these chromosomal conjugative elements, also called conjugative transposons, that the tetracycline resistance genes, tetQ, of Bacteroides are coded, which also exert ribosomal protection [21]. These conjugative transposons normally reside on the chromosome; however, as they have specific int and tra genes, they can excise from there and be transferred to another cell by a conjugative process [23]. The fourth important antibiotic resistance mechanism of Bacteroides strains is against nitroimidazoles; this is mediated by chromosomal or plasmid coded determinants, called nim genes, which are transferable by conjugation [22]. The products of these genes are nitroimidazole reductases [24]. For resistance to carbapenems and nitroimidazoles, the resistance genes should be activated by strong promoters carried on insertion sequence (IS) elements $[10,18,24]$. The cepA genes can be rendered more expressed by some IS elements [24], and the ermF genes may also be upregulated by the promoter sequences of the IS element of the regular transposon [24].

It is important to follow the changes in antibiotic resistance of Bacteroides and to look for genetic elements of known resistance among clinical and normal flora isolates in order to select the best treatment for the severe mixed infections they cause.

\section{MATERIALS AND METHODS}

\section{Bacteria and determination of levels of antibiotic resistance}

Bacteria belonging in the $B$. fragilis group were isolated from severe infections and were identified by conventional tests according to the guidelines of the Manual of Clinical Microbiology [11] and the Wadsworth Anaerobic Bacteriology Manual [25] 
or by the ATB (bioMerieux) system. B. fragilis ATCC 25285 or B. thetaiotaomicron ATCC 29741 was used as control organism in the susceptibility tests.

Antibiotic resistance surveillances were carried out in 1987 (100 B. fragilis group isolates originating from one centre), in 1992 (200 B. fragilis group isolates from three centres), in 1994 (195 B. fragilis group isolates from one centre) and in 1997 (78 B. fragilis group isolates from six centres). The susceptibilities of these isolates were determined by the agar dilution method, by the micro-broth dilution method as recommended by the National Committee for Clinical Laboratory Standards (NCCLS) [15] and by the newly introduced Etest (AB Biodisc, Solna, Sweden), this latter method being carried out according to the prescription of the manufacturer. MIC values were read after incubation for $48 \mathrm{~h}$ at $37{ }^{\circ} \mathrm{C}$ in an anaerobic chamber (Bactron, Shell Lab.).

For PCR detection of the $c f i A$ and nim genes, 242 B. fragilis group isolates were selected in 1998, 1999 and 2000. The following control strains were used: B. fragilis TAL3636 (metallo- $\beta$-lactamase producer), B. fragilis NCTC9344 (carbapenem-sensitive), B. fragilis BF8 (nimA-positive) and B. fragilis 638R (nim-negative).

\section{Detection of $\beta$-lactamase and specific imipenemase activities}

$\beta$-Lactamase activity was determined quantitatively with nitrocefin $(0.10 \mathrm{mM})$ in 50 $\mathrm{mM}$ sodium phosphate buffer $\left(\mathrm{pH} 7.0,37^{\circ} \mathrm{C}\right)$ by a spectrophotometric method [13]. One unit of $\beta$-lactamase was defined as the amount which formed $1.0 \mu$ mole of product per minute under the given conditions. The specific imipenemase activities were determined by following the change in absorbance at $299 \mathrm{~nm}$ of a mixture of bacterial sonicate $(0.2 \mathrm{ml})$, imipenem $(0.2 \mathrm{ml}, 250 \mathrm{mg} / \mathrm{l})$ and phosphate buffer $(\mathrm{pH} 7,0.6$ $\mathrm{ml}$ ) and the results were given in nmole hydrolysed imipenem $/ \mathrm{mg}$ protein $/ \mathrm{min}$.

\section{PCR detection of imipenem and metronidazole resistance genes, and examination of their upregulating IS elements}

Template DNA was prepared by boiling of $0.5 \mathrm{McFarland}$ turbidity solutions of the examined isolates for $10 \mathrm{~min}$, or by the method of Wilson [28] for the nim gene. CfiA PCR reactions were performed on $5 \mu$ of $10 \times$ PCR reaction buffer (Sigma or USB), $1 \mu \mathrm{l}(2.5 \mathrm{mM})$ each of dATP, dCTP, dGTP and dTTP, $1 \mu \mathrm{l}$ (35 pmole) each of the primers, $5 \mu \mathrm{l}$ of DNA template and $33 \mu \mathrm{l}$ of sterile water [7]. The primers and the amplification cycles were the same as described by Podglajen et al. [17]. In the IS element (IS1186, IS1168, IS1169, IS1170, IS4351 and IS942)-specific PCRs, we used the same reaction and amplification conditions as above, except that the sequence of the primers was as described in [10, 18, 20, 26], and annealing was for $1 \mathrm{~min}$ at $48^{\circ} \mathrm{C}$. In the PCR amplifying upstream region of the $c f i A$ gene, the reaction and amplification conditions were the same as above, but oligonucleotide $\mathrm{G}$ [18] and the complementary sequence 565-598 within the $c f i A$ gene (UP2) were used as 
primers, the annealing was at $52{ }^{\circ} \mathrm{C}$ and the elongation lasted for 3 min. For the PCR mapping of the IS element, the upstream primer of the IS942 detection and UP2 and the cfiA downstream primer were used, with the same reaction and amplification conditions as above, but for elongation 3 min was applied at $72{ }^{\circ} \mathrm{C}$.

The PCR for the nim gene detection was carried out as described by Trinh and Reysset [27]. The IS elements were detected as above, and the PCR mapping of the upregulating IS element was performed similarly, using the appropriate primer pair and the same reaction and amplification conditions as described for cfiA.

The amplification products were analysed by electrophoresis in $0.8-1.2 \%$ agarose gels containing $0.5 \mu \mathrm{g} / \mathrm{ml}$ ethidium bromide in TBE buffer ( $89 \mathrm{mM}$ Tris base, $89 \mathrm{mM}$ boric acid, 2 mM EDTA, pH 8.25) at $80 \mathrm{~V}$ constant voltage, together with $100 \mathrm{bp}$ ladder (Sigma) or the PstI digest of $\lambda$ phage DNA as molecular weight markers, and were visualized under UV light.

\section{RESULTS}

\section{Antibiotic resistance surveillances of $\mathrm{B}$. fragilis group isolates}

As antibiotic therapy in severe anaerobic infections is mainly empirical, it is necessary to follow the resistance levels to different antimicrobial agents of the most prevalent anaerobic pathogens from time to time. During surveillances carried out in our laboratories in 1987, 1992, 1994 and 1997, examinations were made of the resistance of $B$. fragilis group isolates to 8 antibiotics used most frequently in the empirical therapy of anaerobic infections (Table 1). Most of these studies were carried out with isolates originating from different centres in Hungary (1992, 1994 and 1997) or isolated in Szeged (1987). All isolates tested were susceptible to imipenem, metronidazole and chloramphenicol; very few isolates $(2.5 \%)$ displayed resistance to amox-

Table 1

Prevalence of $B$. fragilis group isolates resistant to different antibiotics in Hungary in the indicated years

\begin{tabular}{|c|c|c|c|c|}
\hline \multirow{2}{*}{ Antibiotic } & \multicolumn{4}{|c|}{ Percentages of resistant isolates in the following years } \\
\hline & 1987 & 1992 & 1994 & 1997 \\
\hline Ampicillin & 88 & 97 & 98 & 98 \\
\hline Amoxicillin/clavulanic acid & n.t & 2.5 & 2.5 & 2.5 \\
\hline Cefoxitin & 6 & 11 & 11 & 16 \\
\hline Imipenem & n.t. & 0 & 0 & 0 \\
\hline Tetracycline & 53 & 65 & 65 & n.t. \\
\hline Clindamycin & 27 & 23 & 21 & 18 \\
\hline Chloramphenicol & 0 & 0 & 0.5 & 0.5 \\
\hline Metronidazole & 0 & 0 & 0 & 0 \\
\hline
\end{tabular}

n.t. - not tested 
icillin/clavulanic acid. The resistance to ampicillin and tetracycline throughout these years was high (88-98\% and 53-65\%, respectively). Between 1987 and 1997, the resistance to cefoxitin increased from $6 \%$ to $16 \%$. In contrast, the clindamycin resistance of the B. fragilis group isolates decreased during this period, being $27 \%$ in 1987 and $18 \%$ in 1997.

\section{Screening for prevalence of resistance genes among B. fragilis group clinical isolates}

Although no expressed resistance to carbapenems and metronidazole was detected during the resistance surveillances between 1987 and 1997, we were interested in whether the presence of the resistance genes, which could be 'silent' or expressed, can be proven among recently selected isolates.

During the period 1998-2000, a total of 242 Bacteroides isolates were studied by PCR for the presence of the carbapenem resistance determinant, the $c f i A$ gene. Two B. fragilis isolates were included (isolated in 2000) which were found to be highly resistant to imipenem (MICs $>256 \mathrm{mg} / \mathrm{ml}$ ), both being positive for the cfiA gene. Five other isolates that were sensitive to imipenem (MICs $<0.5 \mu \mathrm{g} / \mathrm{ml}$ ) also harboured the cfiA gene (Table 2, Fig. 1).

The $\beta$-lactamase and specific imipenemase productions of the cfiA-positive isolates were determined (Table 2). Four of 5 isolates with low imipenem MICs produced low amounts of $\beta$-lactamases and had low MIC values, and the 2 resistant isolates produced high amounts of carbapenemase and had high imipenem and meropenem MICs. The activation mechanism of carbapenemase production was examined by looking for IS elements upstream of the cfiA gene in these isolates, to prove that such mechanisms are behind the upregulation of our carbapenem-resistant

Table 2

Carbapenem and metronidazole resistance levels, $\beta$-lactamase activities and cfiA and nim gene positivity of selected isolates

\begin{tabular}{|c|c|c|c|c|c|c|}
\hline \multirow{2}{*}{ Isolate } & \multicolumn{3}{|c|}{$\mathrm{MIC}(\mathrm{mg} / \mathrm{ml})$ of } & \multirow{2}{*}{$\begin{array}{c}\beta \text {-Lactamase } \\
\text { activity }(\mathrm{U} / \mathrm{ml})\end{array}$} & \multicolumn{2}{|c|}{ Presence of } \\
\hline & imipenem & meropenem & metronidazole & & $c f i A$ & nim \\
\hline B. fragilis 22 & 0.06 & 0.125 & 0.125 & 0.0175 & + & - \\
\hline B. fragilis 20 & 0.06 & 0.06 & 0.125 & 0.0028 & + & - \\
\hline B. fragilis 98 & 0.125 & 0.25 & 0.047 & 0.0004 & + & - \\
\hline B. fragilis 72 & 0.25 & 0.25 & 0.032 & 0.0109 & + & - \\
\hline B. fragilis $9259 / 5$ & $>256$ & $>32$ & 3 & $37.03 *$ & + & - \\
\hline B. fragilis $515 / 2$ & $>256$ & $>32$ & 4 & $21.60 *$ & + & - \\
\hline B. fragilis 19924 & 0.032 & 0.047 & 12 & n.t. & - & + \\
\hline B. fragilis $29877 / 1$ & 0.125 & 1.5 & 4 & n.t. & + & + \\
\hline
\end{tabular}

n.t. - not tested

*Specific imipenemase activities expresed in nmole hydrolysed imipenem/mg protein $/ \mathrm{min}$. 


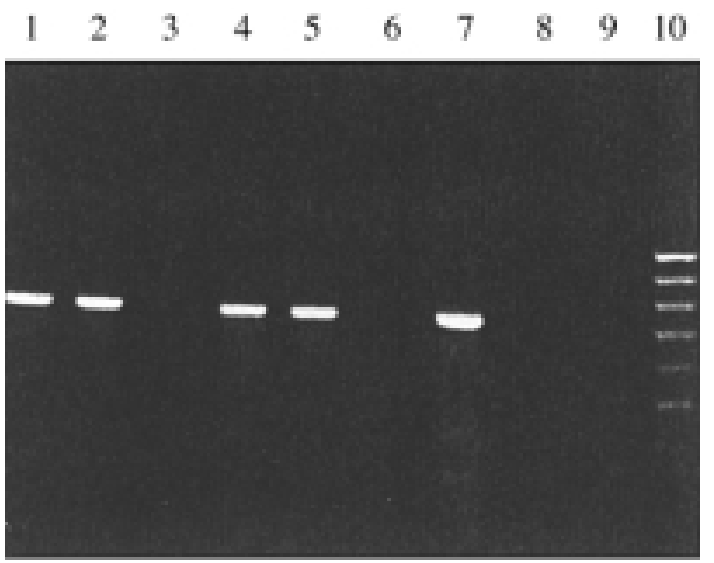

Fig. 1. CfiA PCR of the 'silent' cfiA-positive B. fragilis isolates. Lanes 1-8: B. fragilis isolates 22, 20, 92, 72, 98, 66, TAL3636 (positive control) and NCTC9344 (negative control), respectively. Lane 9: Reagent control. Lane 10: 100 bp ladder as molecular weight marker

isolates. The amplification of the regions upstream of the $c f i A$ genes showed that no insertion occurred for the 5 sensitive isolates harbouring the 'silent' cfiA genes (amplification products of ca. $300 \mathrm{bp}$ found), but in case of the 2 resistant isolates the ca. $1.8 \mathrm{~kb}$ products demonstrated the insertion of some $1.5 \mathrm{~kb}$ DNA segments. The presence of 5 IS elements (IS942, IS1186, IS4351, IS1169 and IS1170) in the genome of the cfiA-positive isolates was also investigated (Figs 2 and 3). The IS942 element was found in the 2 resistant isolates, but no IS element was detected by PCR in 4 of the 5 sensitive strains (the exception was B. fragilis 29877/1, which is moderately metronidazole-resistant and contains IS1186; see later). The PCR mapping of
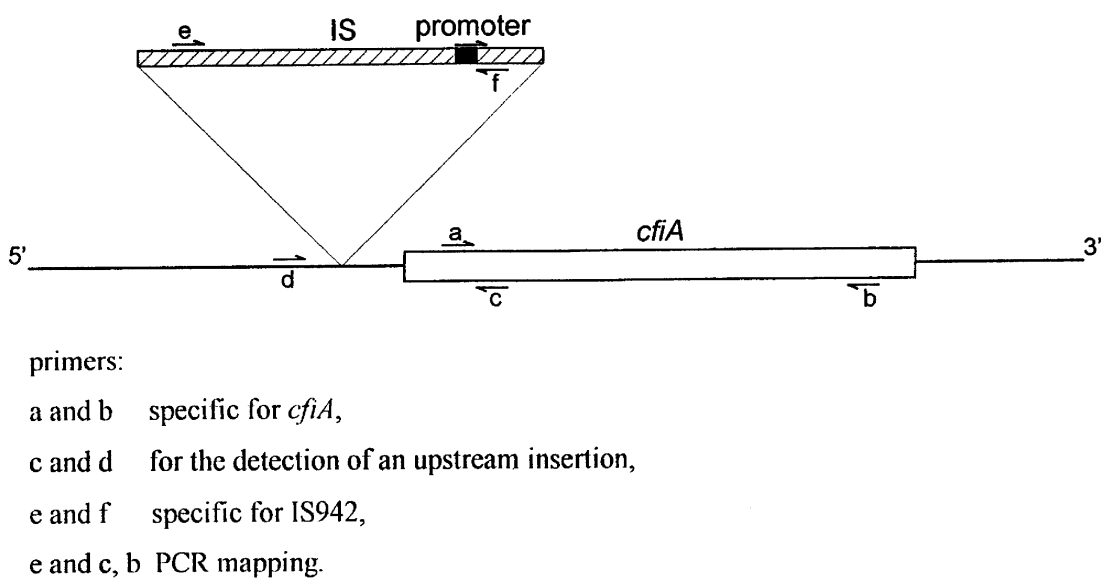

Fig. 2. Strategy of detection of IS elements upstream of cfiA 


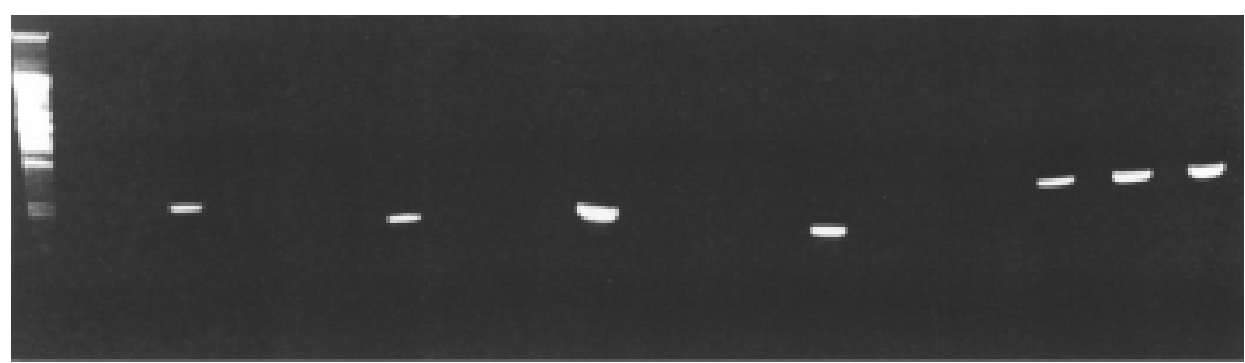

a b
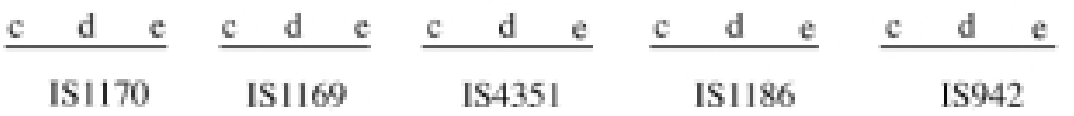

Fig. 3. Detection of 5 IS elements in the 2 imipenem-resistant isolates. The examination of the IS elements is indicated below the picture. a: PstI fragments of $\lambda$ phage DNA as molecular weight marker. b: Reagent control. c: Positive controls for the indicated IS elements. d: B. fragilis 515/2. e: B. fragilis $9259 / 5$

the IS942 elements proved that the $c f i A$ genes could be upregulated by close insertion and correct orientation of the IS942 elements and by their outward-oriented promoters (Fig. 2).

Moderately metronidazole-resistant isolates could also be raised by the insertion of IS elements upstream of 'silent', chromosomally or plasmid located nim genes [22]. Two moderately metronidazole-resistant $B$. fragilis isolates were found in our laboratory during the first few months of 2000, with MIC values of 12 and $4 \mu \mathrm{g} / \mathrm{ml}$ (Table 2). One of the 2 strains carried a 'silent' cfiA gene as well. The nim PCR of these strains demonstrated a $458 \mathrm{bp} \mathrm{nim}$-specific PCR product (Fig. 4). The 2 isolates were

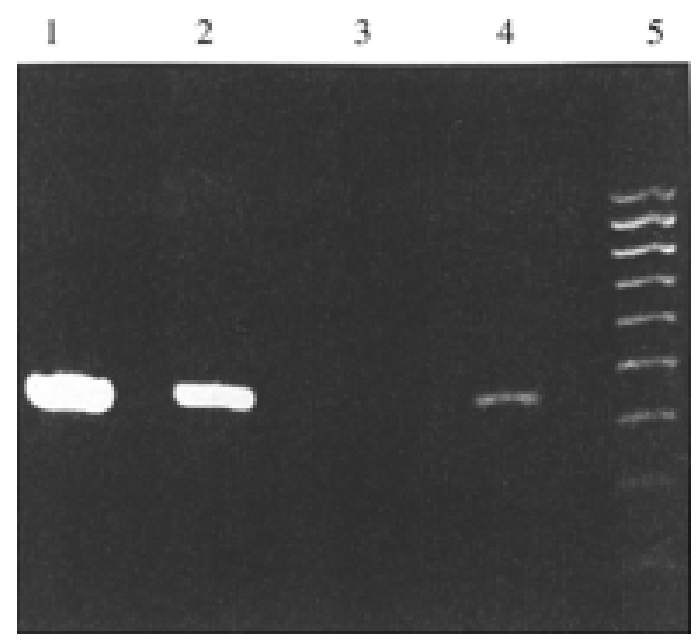

Fig. 4. Nim PCR of the moderately metronidazole-resistant B. fragilis isolates. Lanes 1-4: B. fragilis strains 19924, 29577, 638R (negative control) and BF8 (positive control), respectively. Lane 5: $100 \mathrm{bp}$ ladder as molecular weight marker 
analysed for the presence of IS elements, as in the case of the imipenem-resistant isolates. The presence of the IS1168/IS1186 element was found (these are highly homologous IS elements) in the 2 moderately resistant isolates. The PCR mapping of the IS1168 and IS1186 upstream of the nim gene proved that the nim genes of these latter isolates are activated by the close proximity and the correct orientation of one copy of the IS1168/IS1186 element. No further nim-positive isolates were found by PCR among the 242 metronidazole-sensitive (MICs $<2 \mu \mathrm{g} / \mathrm{ml}$ ) Bacteroides isolates.

\section{DISCUSSION}

Until several years ago, the susceptibility patterns of human pathogenic anaerobic bacteria were relatively stable and predictable. However, in some parts of the world resistance to tetracyclin, penicillin, ampicillin and erythromycin among Bacteroides isolates was already observed during the 1970s, and to cefoxitin and clindamycin in the 1980s. These observations and the shifting susceptibility patterns and variable efficacy of many other $\beta$-lactam antibiotics, especially the newer cephalosporins, against certain anaerobic bacteria, have made considerations concerning screening of the antibiotic susceptibilities of different groups of anaerobic bacteria mandatory [2]. Reports of differences in rates of resistance between countries and hospitals [16] revealed the great importance of monitoring trends in susceptibility from time to time. A comparison of our surveillance data with those obtained during a European study involving 15 countries showed that the $B$. fragilis group isolates in Hungary have the same resistance levels to cefoxitin and clindamycin as in Spain, a country where antibiotics are used with less control as compared with northern European countries [16]. The decrease observed in the clindamycin resistance of B. fragilis group strains between 1987 and 1997 demands further evaluation.

Between 1987 and 1997, similarly as in many European countries [16], no imipenem and metronidazole-resistant Bacteroides isolates were found in Hungary. The first imipenem-resistant $B$. fragilis isolate, producing a special metallo- $\beta$-lactamase, was reported in the USA in 1986 [5] and subsequent reports from other countries confirmed the sporadic existence [7, 21, 29]. The emergence of Bacteroides isolates resistant to metronidazole was first noted in France [3], and later in England [1], demonstrating that such strains may well occur elsewhere, too. It has been shown that specific events are needed for the expression of such resistances; IS elements carrying outward-oriented promoters should be inserted upstream of the 'silent' resistance genes ( $c f i A$ or $\mathrm{nim}$ ), and the prevalence of these 'silent' genes and the activating IS elements in the population should be 'high' enough. The frequency of 'silent' and phenotypically imipenem-resistant Bacteroides isolates seems to be low in countries where the prevalence of the $c f i A$ gene has been examined. In Hungary, the prevalence of 'silent' cfiA-positive and real imipenem-resistant isolates was 2.0 and $0.8 \%$, respectively. These figures are similar to or slightly lower than the values reported in other countries: in France 1.6 and 0.8\% [19], in Japan 2.4 and 1.2\% [29], and in the UK 6.3 and $0.6 \%$ [7], respectively. Different IS elements were found upstream of the 
cfiA gene. Podglajen et al. found the IS1186 insertions upstream of the cfiA gene [18]. Edwards et al. proved the presence of IS1187 and an element similar to the IS942/IS1170 group in meropenem-resistant strains [8]. In our cfiA-positive carbapenem-resistant isolates, the IS942 was confirmed in the right position upstream of the gene. The 2 metronidazole intermediate-resistant isolates harboured the IS1186/IS1168 upstream of the nim genes, which explains the higher MICs found in this case. No silent nim gene was present among our isolates, which tends to suggest the low frequency of such strains.

Special attention should be paid to the fact that clinical isolates harbouring both the cfiA and the nim gene can be found by PCR among the B. fragilis isolates expressing resistance and causing special therapeutic problems in severe infections.

During this study, we detected the first imipenem or metronidazole-resistant (intermediate resistance) Bacteroides isolates in Hungary. The increasing frequency of the presence of 'silent' genes makes it mandatory not only to continue to survey antibiotic resistance among clinical isolates of these important anaerobic pathogens, but also to screen for the presence of resistance genes among clinical and normal flora isolates.

\section{ACKNOWLEDGEMENTS}

This study was supported by grants from the National Science Research Fund (OTKA), Nos. T016222 and $\mathrm{T} 032385$.

\section{REFERENCES}

1. Brazier, J. S., Stubbs, S. L. J., Duerden, B. I. (1999) Metronidazole resistance among clinical isolates belonging to the Bacteroides fragilis group: time to be concerned? J. Antimicrob. Chemother. 44, $580-581$.

2. Breuil, J., Burnat, C., Patey, O., Dublanchet, A. (1989) Survey of Bacteroides fragilis susceptibility patterns in France. J. Antimicrob. Chemother. 24, 69-75.

3. Breuil, J., Dublanchet, A., Truffaut, N., Sebald, M. (1989) Transferable 5-nitroimidazole resistance in the Bacteroides fragilis group. Plasmid 21, 151-154.

4. Bush, K., Jacoby, G. A., Medeiros, A. A. (1995) A functional classification scheme for $\beta$-lactamases and its correlation with molecular structure. Antimicrob. Agents and Chemother. 39, 1211-1233.

5. Cuchural, G. J., Malamy, M. H., Tally, F. P. (1986) $\beta$-Lactamase mediated imipenem resistance in Bacteroides fragilis. Antimicrob. Agents Chemother. 30, 645-648.

6. Edwards, R. (1997) Resistance to $\beta$-lactam antibiotics in Bacteroides spp. J. Med. Microbiol. 46, 979-986.

7. Edwards, R., Hawkyard, C. V., Garvey, M. T., Greenwood, D. (1999) Prevalence and degree of expression of the carbapenemase gene (cfiA) among clinical isolates of Bacteroides fragilis in Nottingham, UK. J. Antimicrob. Chemother. 43, 273-276.

8. Edwards, R., Read, P. N. Expression of the carbapenemase gene (cfiA) in Bacteroides fragilis. $J$. Antimicrob. Chemother. (in press)

9. Finegold, S. M. (1989) Anaerobic infections in humans. Academic Press, San Diego

10. Haggoud, A., Reysset, G., Azeddoug, H., Sebald, M. (1994) Nucleotide sequence analysis of two 5nitroimidazole resistance determinants from Bacteroides strains and of a new insertion sequence upstream of the two genes. Antimicrob. Agents Chemother. 38, 1047-1051.

11. Hannele, R. J-S., Summanen, P. H., Finegold, S. M. (1995) Bacteroides, Prevotella, Fusobacterium, and other anaerobic Gram-negative bacteria. In: Baron, E. J., Murray, P. R., Pfaller, M. A., Tenover, 
F. C., Yolken, R. H. (ed.) Manual of Clinical Microbiology, 6th edn. ASM Press, Washington, D. C. pp. 603-620.

12. Hofstad, T. (1992) Virulence factors in anaerobic bacteria. Eur. J. Clin. Microbiol. Infect. Dis. 11, 1044-1048.

13. Kesado, T., Lindquist, L., Hedberg, M., Tunér, K., Nord, C. E. (1989) Purification and characterization of a new $\beta$-lactamase from Clostridium butyricum. Antimicrob. Agents Chemother. 33, $1302-1307$.

14. Macfarlane, G. T., Macfarlane, S. (1997) Human colonic microbiota: ecology, physiology and metabolic potential of intestinal bacteria. Scand. J. Gastroenterol. Suppl. 222, 3-9.

15. National Committee for Clinical Laboratory Standards (1993) Methods for antimicrobial susceptibility testing of anaerobic bacteria, 3rd edn.; Approved Standard M11-A3. NCCLS Villanova, PA.

16. Phillips, I., King, A., Nord, C. E., Hoffstedt, B. (1992) Antibiotic susceptibility of the Bacteroides fragilis group. Eur. J. Clin. Microbiol. Infect. Dis. 11, 292-304.

17. Podglajen, I., Breuil, J., Bordon, F., Gutmann, L., Collatz E. (1992) A silent carbapenemase gene in strains of Bacteroides fragilis can be expressed after a one-step mutation. FEMS Microbiology Letters 91, 21-31.

18. Podglajen, I., Breuil, J., Collatz, E. (1994) Insertion of a novel DNA sequence, IS1186, upstream of the silent carbapenenase gene, $c f i A$, promotes expression of carbapenem resistance in clinical isolates of Bacteroides fragilis. Mol. Microbiol. 12, 105-114.

19. Podglajen, I., Breuil, J., Casin, I., Collatz, E. (1995) Genotypic identification of two groups within the species Bacteroides fragilis by ribotyping and by analysis of PCR-generated fragment patterns and insertion sequence content. J. Bacteriol. 177, 5270-5275.

20. Rasmussen, B. A., Kovacs, E. (1991) Identification and DNA sequence of a new Bacteroides fragilis insertion sequence-like element. Plasmid 25, 141-144.

21. Rasmussen, B. A., Bush, K., Tally, F. P. (1993) Antimicrobial resistance in Bacteroides. Clin. Infect. Dis. 16 (Suppl.), S390-400

22. Reysset, G., Haggoud, S., Sebald, M. (1995) Genetics and expression of 5-nitroimidazole resistance in anaerobes. In: Duerden, B. I., Wade, W. G., Brazier, J. S., Eley, A., Wren, B., Hudson, M. (ed.) Antibiotics, anaerobes and clinical infections. Science Reviewers, UK.

23. Salyers, A. A., Shoemaker, N. B. (1995) Conjugative transposons: The force behind the spread of antibiotic resistance genes among Bacteroides clinical isolates. Anaerobe 1, 143-150.

24. Smith, C. J., Tribble, G. D., Bayley, D. P. (1998) Genetic elements of Bacteroides species: A moving story. Plasmid 40, 12-29.

25. Summanen, P., Baron, E. J., Citron, D. M., Wexler, H. M., Finegold, S. M. (1993) Wadsworth Anaerobic Bacteriology Manual, 5th edn. Star, Belmont, CA.

26. Trinh, S., Haggoud, A., Reysset, G., Sebald, M. (1995) Plasmids pIP419 and pIP421 from Bacteroides: 5-nitroimidazole resistance genes and their upstream insertion sequence elements. Microbiology 141, 927-935.

27. Trinh, S., Reysset, G. (1996) Detection by PCR of the nim genes encoding 5-nitroimidazole resistance in Bacteroides spp. J. Clin. Microbiol. 34, 2078-2084.

28. Wilson, K. (1994) Miniprep of bacterial genomic DNA. In: Ausubel, F. M., Brent, R., Kingston, R. E., Moore, D. D., Seidman, J. G., Smith, J. A., Struhl, K. (ed.) Current Protocols in Molecular Biology. John Wiley and Sons, Inc., New York, pp. 2.4.1-2.4.2.

29. Yamazoe, K., Kato, N., Kato, H., Tanaka, K., Katagiri, Y., Watanabe, K. (1999) Distribution of the cfiA gene among Bacteroides fragilis strains in Japan and relatedness of cfiA to imipenem resistance. Antimicrob. Agents Chemother. 43, 2808-2810. 